\title{
Influencia del sustrato en la composición de la ictiofauna en zonas someras de la laguna costera Barra de Navidad, México
}

Yureidy Cabrera-Paez ${ }^{1,2}$, Consuelo M. Aguilar-Betancourt ${ }^{2,3 *}$ \& Gaspar González-Sansón ${ }^{2,3}$

1. Programa de Doctorado en Biosistemática, Ecología y Manejo de Recursos Naturales (BEMARENA), Universidad de Guadalajara, Ave. Juárez No. 976, Colonia Centro, Guadalajara, Jalisco, México; yurecab06@yahoo.com

2. Departamento de Estudios para el Desarrollo Sustentable de la Zona Costera, Universidad de Guadalajara, Gómez Farías 82, San Patricio-Melaque, Cihuatlán, Guadalajara, Jalisco, México; coquiaguilar06@yahoo.es, gaspargonzalez2001@yahoo.es

3. Canadian Rivers Institute, 100 Tucker Park Rd, Saint John, New Branswick, Canada.

* Correspondencia

Recibido 03-VI-2020. Corregido 21-VIII-2020. Aceptado 09-IX-2020.

\begin{abstract}
Substrate influence on ichthyofaunal composition in shallow areas of Barra de Navidad coastal lagoon, Mexico. Introduction: Coastal lagoons are important habitats for coastal fishes playing an essential role as nursery areas for many species. While the effects of salinity and temperature on fish abundance spatial and temporal variation have been well documented, research on the influence of substrate characteristics is scarce. Objective: To investigate the correlation of salinity, temperature and sediment characteristics with spatial and temporal variations of the ichthyofauna composition in Barra de Navidad coastal lagoon's shallow zones. Methods: Samples of ichthyofauna were taken with a beach purse seine along a period of two years in four sites. Water salinity and temperature were measured simultaneously with fish sampling operations. Sediment particle size and organic matter content were determined four times at each site during the sampling period. Multivariate methods were used for data analyses. Results: A total of 13487 fish pertaining to 69 species were collected, but only five taxa (Eucinostomus currani, Mugil setosus, Anchoa sp., Diapterus brevirostris, Centropomus robalito) made up more than $80 \%$ of the individuals. Significant differences among all sampling sites and between seasons were found for the ichthyofauna composition. Redundancy analysis showed that changes in ichthyofaunal composition were mainly related to the percentage of mud and organic matter in sediments expressing a spatial gradient. The influence of salinity and temperature was also significant, but these variables explained a smaller proportion of the observed variability and were related mainly to seasonal changes. Conclusions: Sediment particle size and organic matter content are the most important abiotic factors influencing spatial changes in shallow water ichthyofaunal composition. Seasonal changes were correlated with changes in salinity and temperature but could also be related to biological processes such as reproduction and recruitment.
\end{abstract}

Key words: juvenile fish; estuarine system; seasonal variability; spatial variability; Tropical Eastern Pacific; multivariate analysis.

Cabrera-Paez, Y., Aguilar-Betancourt, C.M., \& González-Sansón, G. (2020). Influencia del sustrato en la composición de la ictiofauna en zonas someras de la laguna costera Barra de Navidad, México. Revista de Biología Tropical, 68(4), 1322-1336.

Las lagunas costeras son sistemas estuarinos importantes para el desarrollo del ciclo de vida de los peces costeros y una de sus funciones principales es servir como áreas de crianza para los peces juveniles de muchas especies, una parte de las cuales tienen importancia comercial (Franco et al., 2006). La presencia de peces juveniles en las lagunas costeras en la región del Pacífico de México ha sido documentada por numerosos autores (Amezcua, Madrid-Vera, \& Aguirre-Villaseñor, 2006; Díaz-Ruiz, Aguirre-León, \& Cano-Quiroga, 
2006; Mendoza, Castillo-Rivera, Zárate-Hernández, \& Ortiz-Burgos, 2009; RodríguezRomero et al., 2011) pero en general, no hay mucha información cuantitativa sobre las tallas de los peces examinados que permita conocer la proporción de estadios inmaduros en la ictiofauna. Una excepción importante es el trabajo de González-Sansón et al., (2014), que presentó información detallada sobre la composición por especies y las tallas de los individuos recolectados y concluyó que la ictiofauna de la laguna Barra de Navidad (Jalisco) está compuesta por especies que se encuentran mayormente en estadio juvenil (> $70 \%)$.

La correlación entre variables abióticas (p.e. salinidad, temperatura) y la composición de la ictiofauna ha sido investigada en sistemas estuarinos a nivel mundial (Barletta, Barletta-Bergan, Saint-Paul, \& Hubold, 2005; Franco et al., 2006; Barletta et al., 2008; ReisFilho \& de Alcântara Santos, 2014; Kamrani, Sharifinia, \& Hashemi, 2016). En la costa del Pacífico de México existen también estudios sobre las variaciones espaciales y temporales de la ictiofauna estuarina y su relación con variables ambientales (Castillo-Rivera, Zárate, \& Ortiz, 2005; González-Acosta, De la CruzAgüero, De la Cruz-Agüero, \& Ruiz-Campos, 2005; Castillo-Rivera, Ortiz-Burgos, \& Zárate-Hernández, 2011; Mendoza et al., 2009; Rodríguez-Romero et al., 2011; Aguirre-León, Pérez-Ponce, \& Díaz-Ruiz, 2014). Es notoria, sin embargo, la muy escasa información existente sobre la influencia de las características del sedimento en la variación espacial de los peces estuarinos. Investigaciones recientes realizadas sobre la ictiofauna demersal de zonas profundas en la laguna Barra de Navidad permitieron encontrar evidencia de una alta correlación entre la distribución espacial de los peces y las características del sustrato (Cabrera-Páez, Aguilar-Betancourt, González-Sansón, Negrete-Rodríguez, \& Gray, 2018; GonzálezSansón, Aguilar-Betancourt, \& Kosonoy-Aceves, 2018). Por otra parte, la composición por especies y abundancia de los peces, principalmente peces juveniles, que habitan en zonas someras de la laguna, fue objeto de un estudio que se enfocó a los cambios temporales y analizó los efectos de un huracán sobre esa porción de la ictiofauna de la laguna (González-Sansón et al., 2016). Ese estudio encontró también evidencia cualitativa de cambios espaciales asociados a las características diferentes del sedimento en dos sitios de muestreo.

El objetivo principal de este trabajo fue investigar la posible correlación de la salinidad, la temperatura, la granulometría del sedimento y el contenido de materia orgánica del mismo con la composición de la ictiofauna en las zonas someras de la laguna Barra de Navidad. Tres preguntas guiaron nuestro estudio: 1) ¿Existen cambios espaciales y temporales significativos en la composición de la ictiofauna?; 2) ¿Cuáles son los factores abióticos que se pueden correlacionar mejor con esos cambios?; 3) ¿Explican los factores abióticos gran parte de la variación observada o es posible que factores bióticos también estén influyendo en los cambios observados?

\section{MATERIALES Y MÉTODOS}

Área de estudio: La laguna Barra de Navidad (Fig. 1) se ubica en el litoral centro occidental mexicano del Océano Pacífico $\left(19^{\circ} 11^{\prime} 25^{\prime}\right.$ "N \& 104³9'53” W). Es un sistema lagunar euhalino (salinidad media $>30$ ) con comunicación permanente con el mar (Bahía de Navidad) por medio de una boca permanente. Cuenta con $3.76 \mathrm{~km}^{2}$ de superficie de espejo de agua y profundidades típicas que no exceden los dos metros, aunque en algunas zonas específicas, como el canal de entrada, pueden ser de hasta cuatro metros (González-Sansón et al., 2014). En época de secas (noviembre a mayo) la entrada de agua dulce es mínima y la salinidad media equivale a la del mar adyacente. En época de lluvias (junio a octubre) la laguna recibe entradas de agua superficiales desde los ríos Marabasco y Arroyo Seco, que son corrientes de agua estacionales y se unen a una distancia de aproximadamente $600 \mathrm{~m}$ al este del margen oriental de la laguna. En esta etapa, las aguas lagunares adquieren, por periodos breves de tiempo, un carácter mixohalino (0.5-30), 


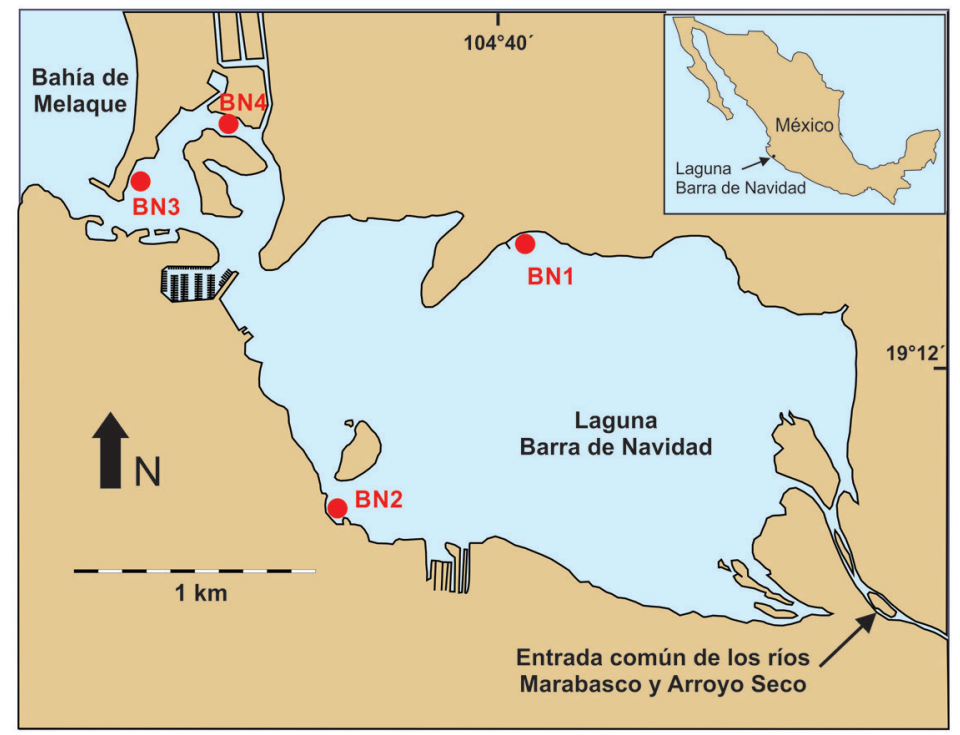

Fig. 1. Laguna Barra de Navidad que muestra la posición de los sitios de muestreo.

Fig. 1. Barra de Navidad lagoon showing the location of sampling sites.

aunque la intensa mezcla por la acción de las mareas restituye rápidamente el carácter euhalino del sistema. En la laguna y sus márgenes existe un desarrollo notable de actividades de pesca artesanal, turísticas y recreacionales.

Variables abióticas: Simultáneamente con los muestreos de la ictiofauna, que se describen a continuación, se midieron los valores de salinidad y temperatura con una sonda YSI-30 previamente a la operación de captura en cada sitio. Al comienzo del estudio, se caracterizó el sedimento en todos los sitios. Para esto se tomaron muestras de sedimento mediante una draga Van Veen, en cuatro meses seleccionados a lo largo de periodo de muestreo que se consideran representativos del sedimento dominante al final de las épocas de lluvia y de seca respectivamente (diciembre de 2015 y 2016, mayo de 2016 y 2017). Se determinó el porcentaje con respecto al peso seco de las fracciones grava $(>2.00 \mathrm{~mm})$, arena $(>0.0625 \mathrm{y} \leq 2.00 \mathrm{~mm})$, lodo $(\leq 0.0625 \mathrm{~mm})$ y materia orgánica según Folk (1974). El contenido de materia orgánica se estimó según Dean (1974), y fue expresado en porcentaje del peso seco total del sedimento.
Muestreo de la ictiofauna: En el periodo comprendido desde septiembre 2015 hasta septiembre 2017, se realizaron muestreos de la ictiofauna en cuatro sitios de la laguna Barra de Navidad (Fig. 1). En cada muestreo se realizaron cinco lances por sitio, empleando un chinchorro playero experimental de $1.75 \mathrm{~m}$ de altura, $10 \mathrm{~m}$ de ancho, $3 \mathrm{~m}$ de largo y $1 \mathrm{~cm}$ de luz de malla en el copo. La captura de los cinco lances fue combinada para obtener una sola muestra en cada sitio y fecha de muestreo. Las recolectas se realizaron mediante arrastres efectuados desde una distancia de $25 \mathrm{~m}$ hasta la orilla, entre las 4:00 pm y las 7:00 pm. Durante el primer año de muestreo las recolectas se realizaron mensualmente. Al cabo de ese tiempo, se hizo un análisis preliminar de los datos obtenidos y este arrojó poca variabilidad en cuanto a los factores abióticos y la ictiofauna entre meses, por lo que se decidió para el segundo año realizar los muestreos en meses alternos.

Los peces capturados en todos los sitios de muestreo fueron guardados en bolsas debidamente rotuladas, conservadas en hielo y transportadas al laboratorio. Posteriormente los individuos se cuantificaron e identificaron 
hasta nivel de especie según Fischer (1995) y Robertson y Allen (2015). Los nombres válidos de las especies se presentan según Fricke, Eschmeyer, y van der Laan (2020) y los nombres y arreglo sistemático de las familias y órdenes según Nelson, Grande, y Wilson (2016). A cada espécimen se le determinó la longitud total $(\mathrm{cm})$ con un ictiómetro. Los ejemplares de mayor talla, como las rayas, fueron fotografiados, medidos in situ y devueltos a la laguna. Se estimó el porcentaje de individuos que estaban por debajo de la longitud de maduración y se consideró que se encontraban en estadio juvenil. La longitud de maduración y el tipo de alimentación de las especies principales incluidas en esta investigación se obtuvieron de la base de datos en línea Fishbase (Froese \& Pauly, 2019) y de literatura científica (Piñón, Amezcua, \& Duncan, 2009; LucanoRamírez, Ruiz-Ramírez, González-Sansón, \& Ceballos-Vázquez, 2014; Aguilar-Betancourt et al., 2016; Ruiz-Ramírez, et al., 2017; Lucano-Ramírez et al., 2019).

Procesamiento estadístico: Para visualizar la tendencia temporal en los valores de salinidad y temperatura, se utilizó el método de suavizado por regresión local (LOESS). Para el análisis de la composición de la ictiofauna se utilizó el procedimiento PERMANOVA (Anderson, 2008) basado en una matriz de afinidades utilizando el índice de similitud de Bray-Curtis calculado sobre la raíz cuarta de los datos del número de individuos para cada especie en cada operación de muestreo. Se definieron como factores de efectos fijos los sitios y las estaciones del año, considerando los meses de muestreo dentro de cada estación como un factor aleatorio anidado en la combinación sitios $\mathrm{x}$ estación. El procesamiento se realizó con el paquete de análisis PRIMER 6.0 (Clarke \& Gorley, 2006).

La relación ambiente-especies fue estimada usando un análisis de redundancia (RDA) (Legendre \& Legendre 2012). Previo al análisis, los datos fueron transformados según lo sugerido por Legendre y Gallagher (2001) para obtener una ordenación basada en distancia de Hellinger. El escalado fue enfocado sobre las correlaciones interespecies, los puntajes (scores) de especies fueron divididas por la desviación estándar y los datos fueron centrados por especies. La significación estadística de los ejes canónicos fue determinada mediante una prueba de Monte Carlo no restringida basada en 9999 permutaciones. En el RDA se incluyeron como variables ambientales los porcentajes de lodo, arena y materia orgánica en el sedimento. El porcentaje de grava en los sedimentos no se incluyó para evitar la colinealidad con las fracciones de lodo y arena. Se incluyeron además la temperatura y la salinidad. Los gráficos conjuntos ("biplots") fueron usados para la representación de los resultados y su interpretación se basó en los criterios de Legendre y Legendre (2012). El procesamiento se realizó con el programa CANOCO 4.5 (ter Braak \& Smilauer, 2002).

\section{RESULTADOS}

Factores abióticos: Los valores medios de salinidad fueron muy similares entre sitios y dentro de cada sitio presentaron poca variación entre estaciones del año. La diferencia fue ligeramente más marcada en los sitios más cercanos a la entrada principal de agua dulce (Tabla 1). Un análisis más detallado de esta variable por meses y sitios permitió verificar disminuciones en los meses finales de la época de lluvias (Fig. 2). En estos meses también se presentó una mayor diferencia entre los valores de salinidad por sitios, sugiriendo la existencia de un gradiente. Estos cambios, sin embargo, fueron de corta duración y una homogeneidad en la salinidad de los sitios se restableció rápidamente. Los valores medios de la temperatura también fueron muy similares entre sitios y resultaron siempre mayores en la época de lluvias en cada sitio, con diferencias estacionales alrededor de 3 a $4{ }^{\circ} \mathrm{C}$ (Tabla 1). El análisis de la variación mensual de los valores de temperatura permitió verificar una tendencia temporal que coincidió bien con la oscilación esperada para esta variable asociada con las estaciones del año (Fig. 2). 
TABLA 1

Valores medios y error estándar (ee) de salinidad, temperatura, fracciones del sedimento y materia orgánica por estaciones en cada sitio de muestreo

TABLE 1

Mean values and standard error (ee) of salinity, temperature $\left({ }^{\circ} \mathrm{C}\right)$, sediment fractions and percentage organic matter by seasons at each sampling site

\begin{tabular}{rlcccccc} 
Sitio & Estación & Salinidad & Temperatura & \% grava & \% arena & \% lodo & \% MO \\
BN1 & lluvia & $28.2 \pm 3.78$ & $33.4 \pm 0.41$ & $1.4 \pm 0.85$ & $16.7 \pm 9.20$ & $81.9 \pm 15.9$ & $8.3 \pm 0.72$ \\
& seca & $31.1 \pm 3.10$ & $30.1 \pm 0.49$ & $0.1 \pm 0.06$ & $2.1 \pm 0.49$ & $97.9 \pm 0.5$ & $17.1 \pm 4.3$ \\
BN2 & lluvia & $29.3 \pm 3.83$ & $31.9 \pm 0.39$ & $8.4 \pm 5.77$ & $13.0 \pm 5.46$ & $78.6 \pm 17.4$ & $9.3 \pm 1.7$ \\
& seca & $31.1 \pm 2.94$ & $28.2 \pm 0.46$ & $0.1 \pm 0.03$ & $1.6 \pm 0.21$ & $98.3 \pm 0.2$ & $18.6 \pm 5.9$ \\
BN3 & lluvia & $32.4 \pm 1.60$ & $31.0 \pm 0.29$ & $25.1 \pm 1.48$ & $73.4 \pm 1.31$ & $1.5 \pm 0.2$ & $1.8 \pm 1.2$ \\
& seca & $31.5 \pm 2.69$ & $27.2 \pm 0.71$ & $3.7 \pm 1.52$ & $83.9 \pm 3.15$ & $12.5 \pm 2.9$ & $1.6 \pm 0.4$ \\
BN4 & lluvia & $29.6 \pm 3.71$ & $31.6 \pm 0.32$ & $17.8 \pm 8.69$ & $73.5 \pm 13.80$ & $8.6 \pm 6.7$ & $1.1 \pm 0.1$ \\
& seca & $30.9 \pm 2.89$ & $28.1 \pm 0.56$ & $16.9 \pm 7.31$ & $80.4 \pm 7.34$ & $2.5 \pm 0.1$ & $0.9 \pm 0.1$ \\
\hline
\end{tabular}
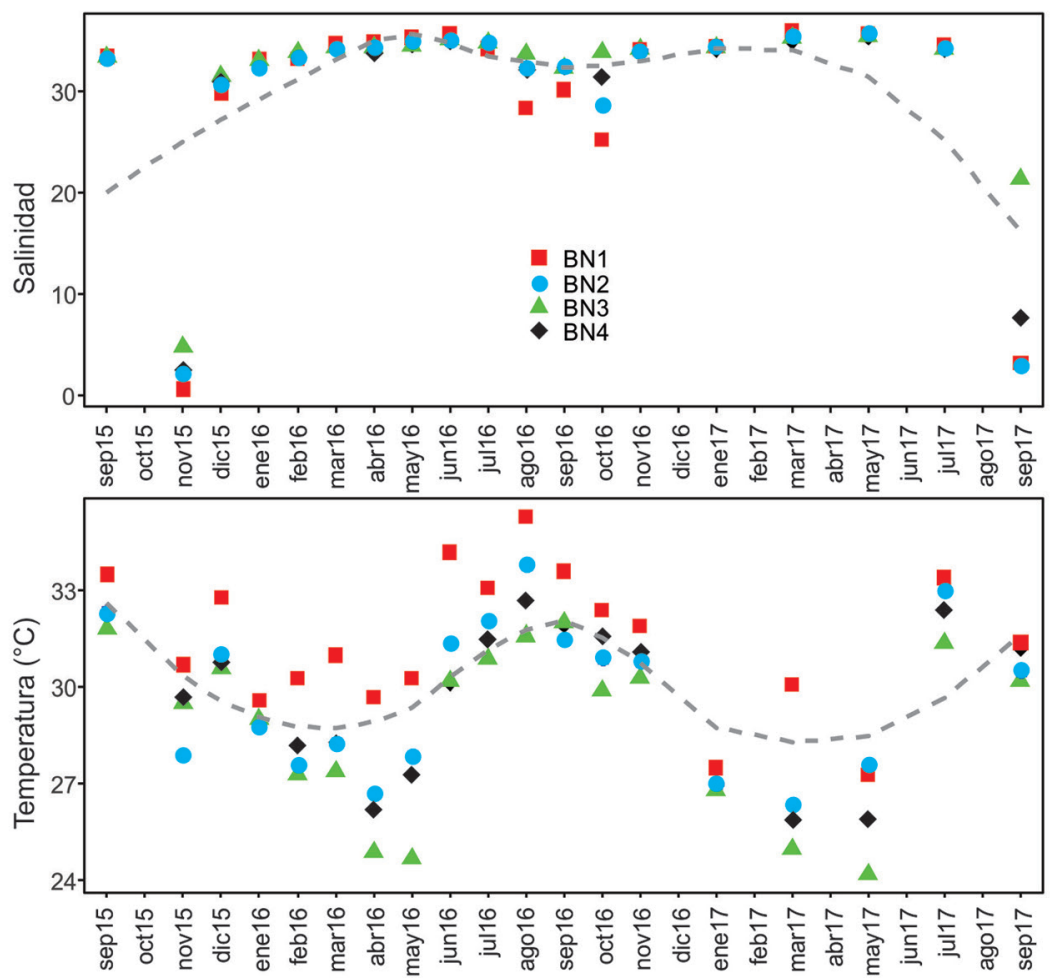

Fig. 2. Valores mensuales por sitios de salinidad y temperatura del agua $\left({ }^{\circ} \mathrm{C}\right)$ en la laguna Barra de Navidad, México. La línea interrumpida indica la tendencia suavizada (LOESS).

Fig. 2. Monthly values by sites of salinity and water temperature $\left({ }^{\circ} \mathrm{C}\right)$ in Barra de Navidad lagoon, Mexico. Dashed line indicates the smoothed trend (LOESS). 
La composición granulométrica del sedimento (Tabla 1) varió notablemente entre los sitios. En BN1 y BN2 predominó ampliamente la fracción lodo (siempre por encima de $78 \%$ ) con fracciones mucho menores de grava y arena. Por el contrario, en los sitios BN3 y BN4, el sedimento estuvo dominado por la arena y en menor medida la grava con fracciones de lodo siempre por debajo del 13 $\%$. Diferencias similares se encontraron para el porciento de materia orgánica, con valores relativamente altos $(>8 \%)$ en $\mathrm{BN} 1$ y BN2 y valores muy bajos (menores de $2 \%$ ) en los sitios BN3 y BN4. En los casos del lodo y la materia orgánica, se observaron valores más bajos en época de lluvia en los sitios BN1 y $\mathrm{BN} 2$, mientras que una tendencia contraria se encontró para la grava y la arena en los mismos sitios (Tabla 1).

\section{Composición general de la ictiofauna:}

Se recolectaron 13487 individuos de 69 especies, pertenecientes a 36 familias, agrupadas en 21 órdenes y dos clases. La familia con mayor representación en número de especies fue Carangidae con ocho especies, seguida de las familias Gerreidae, Gobiidae, Oxudercidae y Lutjanidae con cuatro especies y las familias Tetraodontidae, Eleotridae y Centropomidae con tres especies. El resto de las familias (28) presentaron una o dos especies. Cinco especies (E. currani, M. setosus, Anchoa sp., D. brevirostris, C. robalito) conformaron más del 80 $\%$ de la abundancia y fueron muy dominantes en las capturas. El $98 \%$ de la abundancia total estuvo conformado por 26 especies. En 14 de estas especies, los ejemplares capturados fueron peces juveniles en todos los casos. En otras nueve especies, más del $50 \%$ de los individuos capturados eran peces juveniles. Dos especies presentaron un porciento de adultos por encima del $60 \%$ (P. turrubarensis, Anchoa sp.) y una $(C$. manglicola) apareció como adulto casi en su totalidad. El resto de las especies (43), se presentaron en números muy bajos. Las especies que conformaron más del $98 \%$ de los individuos capturados mostraron hábitos alimentarios que incluyen principalmente el consumo de zooplancton e invertebrados bentónicos, algunas especies además incorporan peces a su dieta y otras se alimentan exclusivamente de detrito (Tabla 2).

TABLA 2

Especies que contribuyen al $98 \%$ de los individuos recolectados, ordenadas según el número medio de individuos capturados por muestreo $(\mathrm{N})$. Código = Etiquetas de especies formadas por las tres primeras letras del género y las tres primeras letras de la especie

TABLE 2

Species contributing $98 \%$ of all collected individuals, ordered after the mean number of individuals by sampling operation $(\mathrm{N})$. Código = Tags for the species formed by three first letters of Genus and three first letters of specific name

\begin{tabular}{lccccccccccc}
\multicolumn{1}{c}{ Especies } & Código & $\mathrm{N}$ & $\%$ & $\%$ acu & LTm & ee & Mín & Máx & LM & $\%<$ LM & A \\
Eucinostomus currani & Euccur & 11.99 & 33.3 & 33.3 & 3.4 & 0.04 & 1.0 & 12.0 & 13.4 & 100 & IB \\
Mugil setosus & Mugset & 7.71 & 21.4 & 54.8 & 7.5 & 0.08 & 2.0 & 20.0 & 23.1 & 100 & D \\
Anchoa sp & Anchoa & 7.60 & 21.1 & 75.9 & 5.3 & 0.06 & 2.2 & 9.5 & 4.7 & 35.7 & ZP \\
Diapterus brevirostris & Diabre & 1.02 & 2.8 & 78.7 & 7.4 & 0.14 & 2.4 & 18.9 & 18.5 & 99.7 & IB \\
Centropomus robalito & Cenrob & 0.77 & 2.1 & 80.9 & 6.4 & 0.21 & 3.4 & 18.7 & 20.9 & 100 & PIB \\
Sphoeroides annulatus & Sphann & 0.70 & 1.9 & 82.8 & 4.6 & 0.24 & 1.0 & 38.0 & 25.9 & 99.6 & IB \\
Lutjanus argentiventris & Lutarg & 0.66 & 1.8 & 84.7 & 8.3 & 0.19 & 2.0 & 17.6 & 32.6 & 100 & PIB \\
Haemulopsis elongatus & Haeelo & 0.58 & 1.6 & 86.3 & 8.0 & 0.16 & 3.0 & 17.5 & 18.5 & 100 & IB \\
Ctenogobius sagittula & Ctesag & 0.53 & 1.5 & 87.8 & 7.0 & 0.23 & 1.8 & 17.5 & 12.9 & 93.9 & D \\
Anchovia macrolepidota & Ancmac & 0.53 & 1.5 & 89.2 & 9.8 & 0.25 & 6.6 & 16.5 & 15.4 & 92.5 & ZP \\
Caranx sexfasciatus & Carsex & 0.37 & 1.0 & 90.3 & 9.4 & 0.20 & 5.0 & 15.5 & 48.4 & 100 & PIB \\
Lutjanus guttatus & Lutgut & 0.33 & 0.9 & 91.2 & 4.2 & 0.05 & 3.7 & 5.6 & 35.1 & 100 & PIB \\
Poeciliopsis turrubarensis & Poetur & 0.28 & 0.8 & 91.9 & 3.7 & 0.08 & 1.2 & 5.5 & 3.1 & 18.6 & ZP \\
\hline
\end{tabular}


TABLA 2 (Continuación) / TABLE 2 (Continued)

\begin{tabular}{lccccccccccc}
\multicolumn{1}{c}{ Especies } & Código & $\mathrm{N}$ & $\%$ & $\%$ acu & LTm & ee & Mín & Máx & LM & $\%<$ LM & A \\
Lutjanus novemfasciatus & Lutnov & 0.28 & 0.8 & 92.7 & 8.8 & 0.36 & 1.8 & 19.6 & 85.6 & 100 & PIB \\
Rhonciscus bayanus & Rhobay & 0.27 & 0.7 & 93.5 & 8.5 & 0.28 & 3.0 & 25.2 & 29.5 & 100 & PIB \\
Syacium ovale & Syaova & 0.26 & 0.7 & 94.2 & 8.6 & 0.62 & 1.9 & 20.3 & 9.5 & 65.6 & PIB \\
Eucinostomus dowii & Eucdow & 0.21 & 0.6 & 94.7 & 5.9 & 0.33 & 1.9 & 13.1 & 12.9 & 98.6 & IB \\
Erotelis armiger & Eroarm & 0.20 & 0.6 & 95.3 & 8.4 & 0.27 & 2.2 & 14.9 & 9.7 & 76.0 & PIB \\
Gobionellus microdon & Gobmic & 0.18 & 0.5 & 95.8 & 7.8 & 0.35 & 2.5 & 13.6 & 8.8 & 61.2 & IB \\
Caranx caninus & Carcan & 0.16 & 0.4 & 96.3 & 9.1 & 0.32 & 5.1 & 16.7 & 54.0 & 100 & PIB \\
Achirus mazatlanus & Achmaz & 0.15 & 0.4 & 96.7 & 10.8 & 0.83 & 1.7 & 20.2 & 10.8 & 54.4 & PIB \\
Oligoplites saurus & Olisau & 0.14 & 0.4 & 97.1 & 7.7 & 0.29 & 2.1 & 12.2 & 21.2 & 100 & PIB \\
Larva leptocefala & Lepto & 0.13 & 0.4 & 97.4 & 3.2 & 0.11 & 1.6 & 6.8 & - & 100 & PIB \\
Centropomus medius & Cenmed & 0.10 & 0.3 & 97.7 & 8.7 & 0.40 & 2.5 & 12.0 & 36.6 & 100 & PIB \\
Ctenogobius manglicola & Cteman & 0.09 & 0.3 & 97.9 & 2.7 & 0.08 & 1.6 & 3.4 & 2.0 & 8.6 & D \\
Gerres simillimus & Gersim & 0.07 & 0.2 & 98.2 & 6.7 & 0.66 & 2.0 & 21.3 & 24.4 & 100 & IB \\
\hline
\end{tabular}

LTm $=$ Longitud total media $(\mathrm{cm})$, ee $=$ error estándar de la LTm, Mín y Máx = Valores mínimos y máximos de la longitud total $(\mathrm{cm}), \mathrm{LM}=$ Longitud de maduración $(\mathrm{cm}), \%<\mathrm{LM}=$ Porciento de individuos con LTm por debajo de la LM, A: Alimentación (IB: Invertebrados bentónicos, D: detrito, PIB: Peces e invertebrados bentónicos, ZP: Zooplancton).

Variaciones espacio-temporales de la ictiofauna: El procedimiento PERMANOVA mostró que la interacción no fue significativa entre los factores sitios y estaciones. Este análisis, además, no arrojó diferencias significativas entre meses para sitios y estaciones (Tabla 3 ). Sin embargo, cada factor (sitios y estaciones) por separado presentó diferencias significativas entre sus niveles. La prueba de comparaciones

TABLA 3

Resultados del PERMANOVA sobre los datos de número de individuos (transformados con raíz cuarta)

TABLE 3

Results of PERMANOVA performed on the number of individuals (fourth root transformed)

\begin{tabular}{lccc}
\multicolumn{1}{c}{ Factor } & GL & Pseudo-F & P \\
Sitios (Si) & 3 & 7.92 & 0.0001 \\
Estaciones (Es) & 1 & 2.42 & 0.0088 \\
Si x Es & 3 & 1.18 & 0.2397 \\
Meses (Si x Es) & 44 & 1.12 & 0.1611 \\
Residual & 23 & & \\
Total & 74 & & \\
\hline
\end{tabular}

$\mathrm{GL}=$ grados de libertad, $\mathrm{P}=$ probabilidad de Pseudo-F basada en permutaciones.

$\mathrm{GL}=$ Degrees of freedom, $\mathrm{P}=$ probability of Pseudo-F based in permutations. múltiples encontró que todos los sitios son diferentes entre sí.

El análisis gráfico de la composición por especies permitió identificar cuáles especies caracterizan a cada sitio en cada estación (Fig. 3). La mayor diversidad de especies y los números de individuos capturados más altos se encontraron en el sitio BN1, mientras que en el sitio BN4 se encontró el número más bajo de especies y en general, el menor número de individuos. En todos los sitios, las especies $E$. currani y $M$. setosus estuvieron bien representadas en ambas estaciones del año. El resto de las especies mostraron diferencias entre sitios más o menos notables. Las especies del género Anchoa estuvieron bien representadas en los sitios BN1 y BN2, pero su representación disminuyó en general en los otros sitios. Las especies S. annulatus, H. elongatus, L. guttatus y $E$. dowii presentaron una clara tendencia a ser más abundantes en los sitios BN3 y BN4. Otras especies estuvieron bien representadas en el sitio BN1, pero fueron escasas en el resto de los sitios. Entre estas se encuentran $C$. robalito, C. sagittula, A. macrolepidota, C. sexfasciatus, $P$. turrubarensis, L. novemfaciatus, $R$. bayanus, $S$. ovale y $E$. armiger. En muchas especies se pudieron verificar diferencias estacionales 


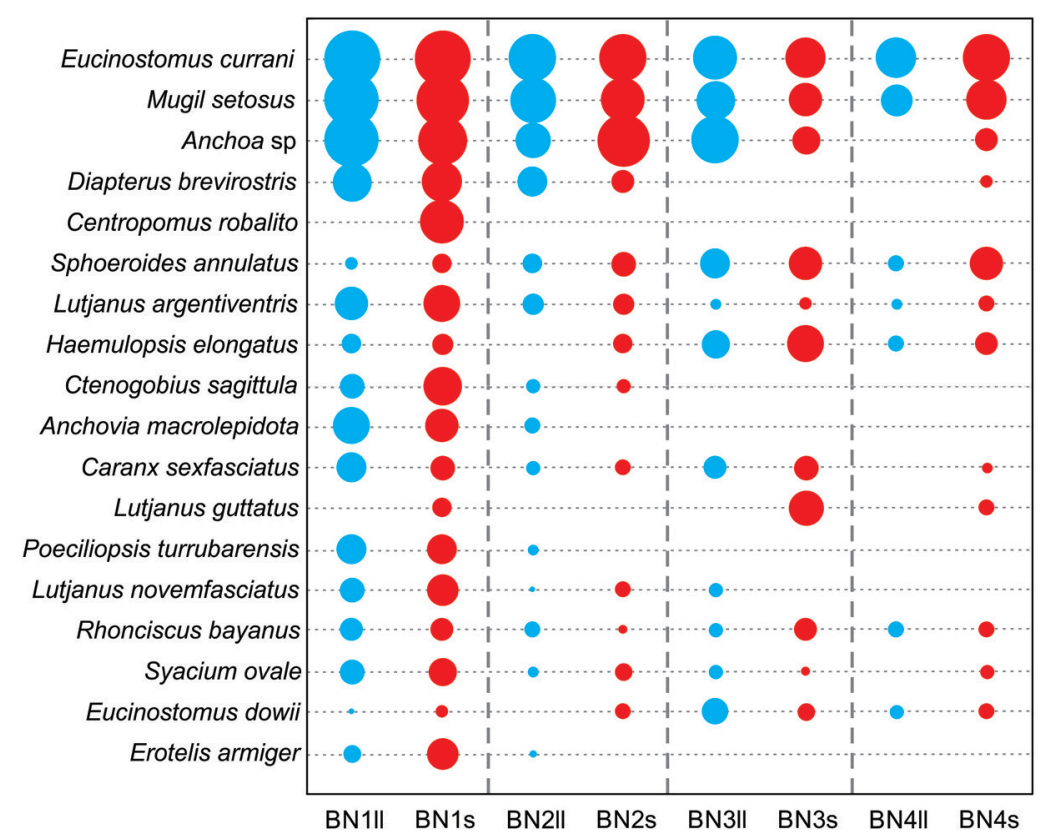

Fig. 3. Composición de la ictiofauna por sitios y estaciones (ll = lluvia; s = seca), en la laguna Barra de Navidad, México. Se muestran las especies más abundantes que suman el $95 \%$ del total de individuos (abreviaturas definidas en la Tabla 2). Los diámetros de los círculos son proporcionales al logaritmo del número de individuos.

Fig. 3. Composition of the ichthyofauna by sites and seasons ( $11=$ rainy; $\mathrm{s}=$ dry) in the Barra de Navidad lagoon, Mexico. Most abundant species adding to $95 \%$ of total individuals are shown (acronyms defined in Table 2). Circles have diameters proportional to logarithm of number of individuals.

en su representación numérica con casos extremos de especies que fueron recolectadas en una estación del año, pero no en la otra.

El modelo RDA ajustado explicó el 18.1\% de la variación total en los datos de especies (Tabla 4). El primer eje canónico fue significativo $(\mathrm{F}=6.928, \mathrm{P}=0.001)$ y explicó el $50.4 \%$ de la relación especies-ambiente. El segundo eje canónico también fue significativo $(\mathrm{F}=3.581, \mathrm{P}=0.025)$ pero explicó un porcentaje más bajo $(22.2 \%)$ de la relación especies-ambiente.

TABLA 4

Análisis de redundancia

TABLE 4

Redundancy analysis

\begin{tabular}{lcccc}
$\quad$ Sumario de la ordenación de los ejes & 1 & 2 & 3 & 4 \\
Valores propios & 0.091 & 0.040 & 0.032 & 0.013 \\
Correlación especies-ambiente & 0.768 & 0.637 & 0.575 & 0.398 \\
Porcentaje de varianza acumulada & & & & 17.6 \\
Datos de especie & 9.1 & 13.1 & 76.3 & 97.5 \\
Relación especies-ambiente & 50.4 & 72.6 & & \\
Suma de todos los valores propios & & & \\
Suma de todos los valores propios canónicos & 1.000 & & \\
\hline
\end{tabular}


El gráfico conjunto ("biplot") de muestras y variables ambientales (Fig. 4) mostró una ordenación de muestras a lo largo del primer eje canónico. Este eje está altamente correlacionado con el porcentaje de lodo y materia orgánica en los sedimentos y se correlaciona negativamente con el porcentaje de arena, lo que se puede interpretar como un gradiente de disminución del tamaño de partículas. La proyección de las muestras sobre este eje permite identificar dos grupos claramente diferenciados de sitios; un grupo está formado por los sitios $\mathrm{BN} 1$ y $\mathrm{BN} 2$ donde predomina un fondo lodoso y un segundo grupo incluye a los sitios $\mathrm{BN} 3$ y $\mathrm{BN} 4$ donde el sustrato es principalmente arenoso (y en algunos casos tiene una fracción importante de grava). Esto demuestra claramente un importante componente espacial de variabilidad. El segundo eje está relacionado positivamente con la salinidad y negativamente con la temperatura. Esto se refleja en el $38.3 \%$ de las muestras durante la temporada de seca y que se encuentran en los cuadrantes superiores. Este eje refleja un segundo gradiente más débil relacionado con el cambio estacional. Un "biplot" de especies y variables ambientales (Fig. 4) permitió definir dos grupos principales de especies. Un primer
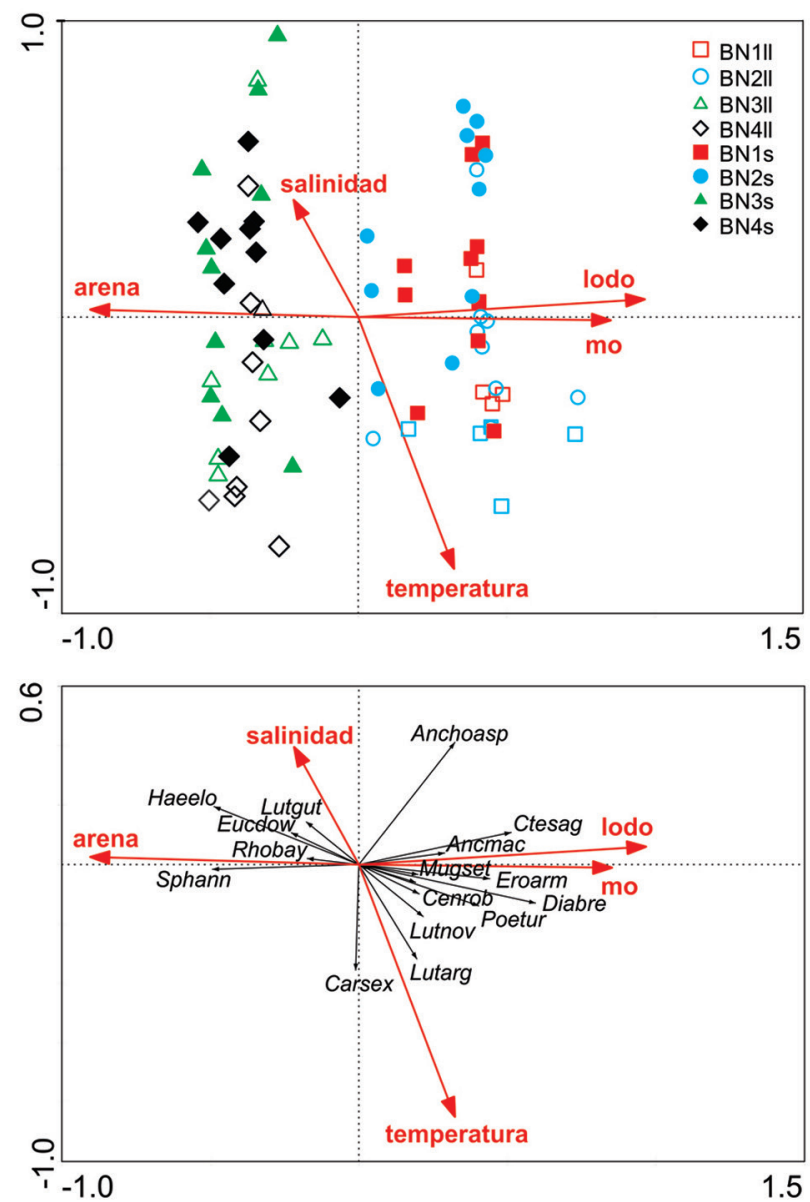

Fig. 4. Gráficos conjuntos de A. variables abióticas con sitios y estaciones ( 11 = lluvia, $\mathrm{s}=$ seca) y $\mathbf{B}$. variables abióticas con especies basados en los resultados del análisis de redundancia, en la laguna Barra de Navidad, México.

Fig. 4. Biplots of $\mathbf{A}$. abiotic variables and sites and seasons ( $11=$ rainy, $\mathbf{s}=$ dry $)$ and $\mathbf{B}$. abiotic variables and species based on the results of redundancy analysis, in the Barra de Navidad lagoon, Mexico. 
grupo altamente correlacionado con el eje principal y que incluyó cuatro de las cinco especies más abundantes (E. currani, M. setosus, D. brevirostris y $C$. robalito). El segundo grupo se correlacionó negativamente con el primer eje e incluyó las especies $H$. elongatus, $S$. annulatus $E$. dowii y $R$. bayanus como las más significativas. Estas especies se presentan con una abundancia relativamente baja en comparación al primer grupo. La especie L. guttatus y la tercera en abundancia, Anchoa sp., mostraron una correlación alta con el segundo eje. Un patrón similar pero correlacionado negativamente con el segundo eje se observa en la especie poco abundante $C$. sexfasciatus.

\section{DISCUSIÓN}

La composición general de la ictiofauna de zonas someras encontrada en el presente estudio coincide bien con lo encontrado por otros autores en sistemas estuarinos de la costa mexicana del Pacífico. Diversos estudios plantean que las especies predominantes en estos ecosistemas variables pertenecen a las familias Gerreidae (Castillo-Rivera et al., 2005; Rodríguez-Romero et al., 2011; Aguirre-León et al., 2014; González-Sansón et al., 2016; Cabrera et al., 2018; Romero-Berny, VelázquezVelázquez, Schmitter-Soto, \& Salgado-Ugarte, 2020); Mugilidae (Cabral-Solis \& Espino-Barr, 2004; Castellanos-Galindo, Krumme, Rubio, \& Saint-Paul, 2013; González-Sansón et al., 2018); Centropomidae (Álvarez-Rubio, Amezcua-Linares, \& Yáñez-Arancibia, 1986; Díaz-Ruiz et al., 2006) y Engraulidae (González-Acosta et al., 2005, Castellanos-Galindo et al., 2013; Padilla-Serrato et al., 2017). Las especies de estas familias presentan un amplio rango de tolerancia a los cambios de salinidad (Mendoza et al., 2009) y tienen una distribución circunglobal, en áreas templadas y tropicales (Nelson et al., 2016).

La laguna Barra de Navidad pudiera considerarse como un hábitat importante de peces juveniles debido al gran número de individuos capturados que presentaban una talla muy por debajo de la longitud de primera maduración reportado en la literatura. La abundancia alta de especies en fase juvenil encontradas en este trabajo refuerza el criterio de la función de área de crianza potencial de este sistema, argumento que ha sido planteado por otros autores para este y otros ecosistemas estuarinos (ÁlvarezRubio et al., 1986; Acevedo, 1997; Nagelkerken et al., 2001; González-Acosta et al., 2005; Amezcua et al., 2006; Rodríguez-Romero et al., 2011; González-Sansón et al., 2014; González-Sansón et al., 2016; Cabrera et al., 2018).

La tendencia al incremento del número de especies y una representación numérica mayor en época de secas, encontrada en esta investigación, pudiera estar asociada a un incremento de la salinidad, que permite el ingreso al sistema de especies marinas más estenohalinas. Por otra parte, la aparición casi exclusiva en el sitio BN1 de especies más afines con salinidades bajas o agua dulce como son C. sagittula, $P$. turrobarensis y E. armiger indica también una influencia de esta variable en la distribución de los peces en este sistema estuarino. Numerosos estudios han encontrado que la salinidad constituye el principal factor responsable de los cambios estacionales y estructuran las comunidades ícticas (Barletta et al., 2005, Faunce \& Serafy, 2006; Sosa-López, Mouillot, RamosMiranda, Flores-Hernandez, \& Chi, 2007; Selleslagh et al., 2009; Rodríguez-Climent, Caiola, \& Ibáñez, 2013; Kamrani et al., 2016). Sin embargo, la variación estacional de la salinidad documentada en nuestra investigación fue moderada y sugiere una limitada influencia de este factor sobre la composición y abundancia de la ictiofauna estuarina, si se tiene en cuenta que la mayoría de los peces que habitan en la laguna Barra de Navidad presentan alta tolerancia a cambios de salinidad.

La temperatura estuvo relacionada inversamente con la salinidad porque la estación de secas coincide con la etapa invernal y la de lluvias con el verano. Por eso es difícil distinguir por separado el efecto de estos factores en la composición de la ictiofauna. Otros estudios han encontrado que la temperatura constituye uno de los factores causantes de los cambios estacionales en las comunidades 
de peces (Acevedo, 1997; González-Acosta et al., 2005; Rodríguez-Romero et al., 2011), pero en la presente investigación, la poca variación de la temperatura media entre estaciones (diferencia alrededor de $4{ }^{\circ} \mathrm{C}$ ) presenta poca relevancia ecológica.

Es común que las comunidades de peces presenten diferencias estacionales en la composición por especies como resultado de los desfases en los tiempos de desove y las migraciones secuenciales de los peces (CastilloRivera et al., 2011). Por tanto, la variabilidad estacional en la composición y abundancia de la ictiofauna presente en este trabajo puede estar influenciada por procesos biológicos estacionales como la reproducción, facilitando el reclutamiento de muchas especies en determinada época del año (Barletta et al., 2008; Selleslagh et al., 2009; Sheaves \& Johnston, 2009). Estos eventos biológicos actúan de forma sinérgica con los factores abióticos y dificultan una interpretación más completa de los resultados obtenidos, por lo que se hace necesario realizar un estudio del ciclo de vida de las especies más abundantes en esta laguna.

Los resultados obtenidos muestran que el tipo de sedimento y el contenido de materia orgánica influyen notablemente en la abundancia y composición por especies de la ictiofauna en la laguna en las zonas someras cerca de la orilla. La influencia del sustrato en la distribución espacial de invertebrados y peces ha sido documentada por varios autores (Anderson, 2008; Selleslagh et al., 2009; RodríguezRomero et al., 2011; Reis-Filho \& de Alcântara Santos, 2014; González-Sansón et al., 2016; González-Sansón et al., 2018). El incremento en número de individuos y abundancia de especies de peces en los sitios donde el sustrato es lodoso y presenta alto por ciento de materia orgánica, pudiera estar estrechamente relacionado con la alimentación; esta tendencia ha sido planteada por Rodríguez-Romero et al., (2011) en una laguna costera en Baja California Sur. Numerosos autores argumentan la alta dependencia de los invertebrados bentónicos al tipo de sustrato y a los nutrientes de origen orgánico (Acevedo, 1997; Vinagre,
Fonseca, Cabral, \& Costa, 2006; Franco et al., 2006; Anderson, 2008; Van der Wal et al, 2008; Alves, Sutherland, \& Gill, 2012, Van Colen et al., 2014). En general, la mayoría de las especies capturadas en esta investigación se alimentan de invertebrados bentónicos y peces pequeños, y estos últimos, a su vez, tienen a los invertebrados pequeños como componente principal en su dieta (Álvarez-Rubio et al., 1986; Arenas-Granados \& Acero, 1992; CruzAgüero, Arellano-Martínez, Cota-Gómez, \& Cruz-Agüero, 1997; Bussing, 1998; FloresOrtega et al, 2015; Padilla-Gutiérrez, GonzálezSansón, Aguilar-Betancourt, \& Flores-Ortega, 2020). Otras especies de peces se alimentan de detrito (González-Sansón et al., 2016). Se puede asumir, por tanto, que la distribución de los peces está respondiendo a la distribución de su fuente principal de alimento. El sustrato podría influir también de otras formas que no están relacionadas directamente con la presencia y abundancia de organismos presas. Tal es el caso de una especie de lenguado ( $S$. ovale), en la cual su distribución espacial podría estar fuertemente relacionada con la conducta de enterrarse en el fondo lodoso para escapar de los depredadores (Selleslagh et al., 2009).

En resumen, la composición granulométrica del sedimento y el contenido de materia orgánica del mismo, constituyen los factores abióticos con mayor influencia en las variaciones espaciales de la composición y abundancia de la ictiofauna en las zonas más someras de la laguna Barra de Navidad. Por otro lado, las variaciones estacionales de la temperatura y salinidad presentan menor influencia. Una parte importante de la variación espacio-temporal no pudo ser explicada por las variables abióticas. Por ese motivo, se puede asumir que las mismas son probablemente el resultado de una combinación de factores ambientales y biológicos actuando simultáneamente. La alta abundancia de peces juveniles demuestra la importancia de este sistema estuarino y de su función como área de crianza potencial en el completamiento del ciclo de vida de muchas de las especies dependientes de este ecosistema. 
Declaración de ética: los autores declaran que todos están de acuerdo con esta publicación y que han hecho aportes que justifican su autoría; que no hay conflicto de interés de ningún tipo; y que han cumplido con todos los requisitos y procedimientos éticos y legales pertinentes. Todas las fuentes de financiamiento se detallan plena y claramente en la sección de agradecimientos. El respectivo documento legal firmado se encuentra en los archivos de la revista.

\section{AGRADECIMIENTOS}

Nuestra gratitud para Armando Alvízar Martínez que ofreció un gran apoyo en los muestreos y para los estudiantes de la Carrera de Biología Marina de la Universidad de Guadalajara que voluntariamente participaron en las actividades de muestreo y procesamiento de muestras. Este trabajo fue financiado parcialmente por una beca para estudios de posgrado del Consejo Nacional de Ciencia y Tecnología de México (CONACyT). La investigación formó parte de un proyecto financiado por la Universidad de Guadalajara.

\section{RESUMEN}

Introducción: Las lagunas costeras son hábitats importantes para los peces costeros y juegan un rol esencial como áreas de crianza para muchas especies. Mientras que los efectos de la salinidad y la temperatura sobre la abundancia de los peces han sido bien documentados, las investigaciones sobre la influencia de las características del sustrato son más escasas. Objetivo: Investigar la correlación de la salinidad, la temperatura y las características del sedimento con las variaciones espaciales y temporales de la composición de la ictiofauna en las zonas someras de la laguna costera Barra de Navidad. Métodos: Se tomaron muestras de la ictiofauna con un chinchorro playero a lo largo de un periodo de dos años en cuatro sitios de la laguna. La salinidad y la temperatura del agua se midieron simultáneamente con las operaciones de muestreo. Se determinaron el tamaño de partículas del sedimento y su contenido de materia orgánica en cuatro momentos a lo largo del periodo de muestreo. Métodos multivariantes fueron utilizados para el análisis de los datos. Resultados: Un total de 13487 peces pertenecientes a 69 especies fueron recolectados, pero solo cinco taxones (Eucinostomus currani, Mugil setosus, Anchoa sp., Diapterus brevirostris,
Centropomus robalito) conformaron más del $80 \%$ de los individuos. Se encontraron diferencias significativas en la composición de la ictiofauna entre todos los sitios de muestreo y entre las estaciones del año. El análisis de redundancia mostró que los cambios en la ictiofauna estuvieron relacionados principalmente con el porcentaje de lodo y el contenido de materia orgánica del sedimento, como expresión de un gradiente espacial. La influencia de la salinidad y la temperatura fue significativa también, pero estas variables explicaron una proporción menor de la variabilidad observada y estuvieron relacionadas principalmente con los cambios estacionales. Conclusiones: El tamaño de las partículas del sedimento y el contenido de materia orgánica del mismo son los factores abióticos más importantes que influyen en los cambios espaciales de la composición de la ictiofauna. Los cambios estacionales estuvieron correlacionados con cambios en la salinidad y la temperatura, pero podrían estar vinculados también a procesos biológicos como la reproducción y el reclutamiento.

Palabras clave: peces juveniles; sistema estuarino; variabilidad estacional; variabilidad espacial; Pacífico Oriental Tropical; análisis multivariante.

\section{REFERENCIAS}

Acevedo, A. (1997). Caracterización ecológica de la comunidad ictica de la Laguna Ojo de Liebre, BCS México (Tesis Maestría). Instituto Politécnico Nacional, Centro Interdisciplinario de Ciencias Marinas, México.

Aguilar-Betancourt, C.M., González-Sansón, G., Kidd, K.A., Munkittrick, K.R., Curry, R.A., KosonoyAceves, D. ... Flores-Ortega, J.R. (2016). Fishes as indicators of untreated sewage contamination in a Mexican coastal lagoon. Marine Pollution Bulletin, 113(1-2), 100-109.

Aguirre-León, A., Pérez-Ponce, H.E., \& Díaz-Ruiz, S. (2014). Heterogeneidad ambiental y su relación con la diversidad y abundancia de la comunidad de peces en un sistema costero del Golfo de México. Revista de Biología Tropical, 62(1), 157-176.

Álvarez-Rubio, M., Amezcua-Linares, F., \& Yáñez-Arancibia, A. (1986). Ecología y estructura de la comunidad de peces en el sistema lagunar Teacapán-Agua Brava, Nayarit, Pacífico central de México. Anales del Instituto de Ciencias del Mar y Limnología, Universidad Nacional Autónoma de México, 13, 185-242.

Alves, J.A., Sutherland, W.J., \& Gill, J.A. (2012). Will improving wastewater treatment impact shorebirds? Effects of sewage discharges on estuarine invertebrates and birds. Animal Conservation, 15(1), 44-52.

Amezcua, F., Madrid-Vera, J., \& Aguirre-Villaseñor, H. (2006). Efecto de la pesca artesanal de camarón sobre la ictiofauna en el sistema lagunar de Santa María 
la Reforma, suroeste del Golfo de California. Ciencias Marinas, 32(1b), 97-109.

Anderson, M.J. (2008). Animal-sediment relationships revisited: Characterising species' distributions along an environmental gradient using canonical analysis and quantile regression splines. Journal of Experimental Marine Biology and Ecology, 366(1-2), 16-27.

Arenas-Granados, P., \& Acero, A. (1992). Organización trófica de las mojarras (Pisces: Gerreidae) de la Ciénaga Grande de Santa Marta (Caribe colombiano). Revista de Biología Tropical, 40(3), 287-302.

Barletta, M., Amaral, C.S., Corrêa, M.F.M., Guebert, F., Dantas, D.V., Lorenzi, L., \& Saint-Paul, U. (2008). Factors affecting seasonal variations in demersal fish assemblages at an ecocline in a tropical-subtropical estuary. Journal of Fish Biology, 73(6), 1314-1336.

Barletta, M., Barletta-Bergan, A., Saint-Paul, U.S.G.H., \& Hubold, G. (2005). The role of salinity in structuring the fish assemblages in a tropical estuary. Journal of Fish Biology, 66(1), 45-72.

Bussing, W. (1998). Gerreidae. Mojarras. In W. Fischer, F. Krupp, W. Schneider, C. Sommer, K. Carpenter, \& V. Niem (Eds.), Guía FAO para identificación de especies para los fines de la pesca. Pacífico CentroOriental (pp. 1114-1128). Roma, Italia: FAO.

Cabral-Solís, E.G., \& Espino-Barr, E. (2004). Distribución y abundancia espacio-temporal de los peces en la laguna de Cuyutlán, Colima, México. CICIMAR Oceánides, 19(1-2), 19-27.

Cabrera-Páez, Y.C., Aguilar-Betancourt, C.M., GonzálezSansón, G., Negrete-Rodríguez, F.N., \& Gray, M. (2018). Sediment granulometry and salinity drive spatial and seasonal variability of an estuarine demersal fish assemblage dominated by juvenile fish. Estuarine, Coastal and Shelf Science, 212, 241-252.

Castellanos-Galindo, G.A., Krumme, U., Rubio, E.A., \& Saint-Paul, U. (2013). Spatial variability of mangrove fish assemblage composition in the tropical eastern Pacific Ocean. Reviews in Fish Biology and Fisheries, 23(1), 69-86.

Castillo-Rivera, M., Ortiz-Burgos, S., \& Zárate-Hernández, R. (2011). Estructura de la comunidad de peces estuarinos en un hábitat con vegetación sumergida: variación estacional y nictémera. Hidrobiológica, 21(3), 311-321.

Castillo-Rivera, M., Zárate, R., \& Ortiz, S. (2005). Variación nictímera y estacional de la abundancia, riqueza y especies dominantes de peces, en un ambiente salobre de sustrato blando. Hidrobiológica, 15(2), 227-238.

Clarke, K., \& Gorley, R. (2006). PRIMER V6: User Manual/ Tutorial. Plymouth, Reino Unido: PRIMER-E.
Cruz-Agüero, J., Arellano-Martínez, M., Cota-Gómez, V., \& Cruz-Agüero, G. (1997). Catálogo de peces marinos de Baja California Sur. México D.F., México: Instituto Politécnico Nacional, Centro Interdisciplinario de Ciencias Marinas, Consejo Nacional para la Biodiversidad.

Dean, W.E. (1974). Determination of carbonate and organic matter in calcareous sediments and sedimentary rocks by loss on ignition; comparison with other methods. Journal of Sedimentary Research, 44(1), 242-248.

Díaz-Ruiz, S., Aguirre-León, A., \& Cano-Quiroga, E. (2006). Evaluación ecológica de las comunidades de peces en dos sistemas lagunares estuarinos del sur de Chiapas, México. Hidrobiológica, 16(2), 197-210.

Faunce, C.H., \& Serafy, J.E. (2006). Mangroves as fish habitat: 50 years of field studies. Marine Ecology Progress Series, 318, 1-18.

Fischer, W. (1995). Guía FAO para la identificación de especies para los fines de la pesca. Pacífico centrooriental: Vertebrados. Roma, Italia: FAO.

Flores-Ortega, J.R., González-Sansón, G., Aguilar-Betancourt, C., Kosonoy-Aceves, D., Venegas-Muñoz, A., Lucano-Ramírez, G., \& Ruiz-Ramírez, S. (2015). Hábitos alimentarios de los jóvenes de Centropomus robalito (Centropomidae: Actinopterygii) en la laguna de Barra de Navidad, Jalisco, México. Revista de Biología Tropical, 63(4), 1071-1081.

Folk, R. (1974). Petrology of sedimentary rocks. Austin, Estados Unidos de América: Hemphill Pub. Co.

Franco, A., Franzoi, P., Malavasi, S., Riccato, F., Torrice1li, P., \& Mainardi, D. (2006). Use of shallow water habitats by fish assemblages in a Mediterranean coastal lagoon. Estuarine, Coastal and Shelf Science, 66(1-2), 67-83.

Fricke, R., Eschmeyer, W., \& van der Laan, R. (2020). Catalog of fishes: genera, species, references. Recuperado de http://researcharchive.calacademy.org/ research/ichthyology/catalog/fishcatmain.asp

Froese, R., \& Pauly, D. (2019). FishBase. World Wide Web electronic publication. Recuperado de www. fishbase.org

González-Acosta, A.F., De la Cruz-Agüero, G., De la Cruz-Agüero, J., \& Ruiz-Campos, G. (2005). Seasonal pattern of the fish assemblage of El Conchalito mangrove swamp, La Paz bay, Baja California Sur, Mexico. Hidrobiológica, 15(2), 205-214.

González-Sansón, G., Aguilar-Betancourt, C., KosonoyAceves, D., Lucano-Ramírez, G., Ruiz-Ramírez, S., Flores-Ortega, J.R., ... Silva-Bátiz, F.D.A. (2014). Composición por especies y tallas de los peces en la laguna Barra de Navidad, Pacífico central mexicano. Revista de Biología Tropical, 62(1), 142-157. 
González-Sansón, G., Aguilar-Betancourt, C., KosonoyAceves, D., Lucano-Ramírez, G., Ruiz-Ramírez, S., \& Flores-Ortega, J.R. (2016). Variaciones espaciales y temporales de la abundancia de peces juveniles en la laguna costera Barra de Navidad, Jalisco, México: Efectos del huracán. Revista de Biología Marina y Oceanografia, 51(1), 123-136.

González-Sansón, G., Aguilar-Betancourt, C.M., \& Kosonoy-Aceves, D. (2018). Influence of sediment granulometry and salinity on the composition of an estuarine fish assemblage in the Mexican Tropical Pacific. Revista de Biología Tropical, 66(3), 1065-1077.

Kamrani, E., Sharifinia, M., \& Hashemi, S.H. (2016). Analyses of fish community structure changes in three subtropical estuaries from the Iranian coastal waters. Marine Biodiversity, 46(3), 561-577.

Legendre, P., \& Gallagher, E.D. (2001). Ecologically meaningful transformations for ordination of species data. Oecologia, 129(2), 271-280.

Legendre, P., \& Legendre, L.F. (2012). Numerical ecology. Ámsterdam, Países Bajos: Elsevier.

Lucano-Ramírez, G., Ruiz-Ramírez, S., González-Sansón, G., \& Ceballos-Vázquez, B.P. (2014). Reproductive biology of the yellow snapper, Lutjanus argentiventris (Pisces, Lutjanidae), from the Mexican central Pacific. Ciencias Marinas, 40(1), 33-44.

Lucano-Ramírez, G., Gómez-García, M., Ruiz-Ramírez, S., González-Sansón, G., Aguilar-Betancourt, C., \& Flores-Ortega, J.R. (2019). Reproductive characteristics of the sole Achirus mazatlanus (Pleuronectiformes: Achiridae) in the Barra de Navidad coastal lagoon, Jalisco, Mexico. Ciencias Marinas, 45(2), 47-58.

Mendoza, E., Castillo-Rivera, M., Zárate-Hernández, R., \& Ortiz-Burgos, S. (2009). Seasonal variations in the diversity, abundance, and composition of species in an estuarine fish community in the Tropical Eastern Pacific, Mexico. Ichthyological Research, 56(4), 330-339.

Nagelkerken, I., Kleijnen, S., Klop, T., Van Den Brand, R.A.C.J., de La Moriniere, E.C., \& Van der Velde, G. (2001). Dependence of Caribbean reef fishes on mangroves and seagrass beds as nursery habitats: a comparison of fish faunas between bays with and without mangroves/seagrass beds. Marine Ecology Progress Series, 214, 225-235.

Nelson, J.S., Grande, T.C., \& Wilson, M.V. (2016). Fishes of the World. New Jersey, USA: John Wiley \& Sons.

Padilla-Gutiérrez, S.C., González-Sansón, G., AguilarBetancourt, C.M., \& Flores-Ortega, J.R. (2020). Comparative analysis of the diet composition and its relation to morphological characteristics in Achirus mazatlanus and Syacium ovale (Pleuronectiformes:
Osteichthyes) from a Mexican Pacific coastal lagoon. Neotropical Ichthyology, 18(1), e190059.

Padilla-Serrato, J., López-Martínez, J., Rodríguez-Romero, J., Acevedo-Cervantes, A., Galván-Magaña, F., \& Lluch-Cota, D. (2017). Changes in fish community structures in a coastal lagoon in the Gulf of California, México. Revista de Biología Marina y Oceanografia, 52(3), 567-579.

Piñón, A., Amezcua, F., \& Duncan, N. (2009). Reproductive cycle of female yellow snapper Lutjanus argentiventris (Pisces, Actinopterygii, Lutjanidae) in the SW Gulf of California: gonadic stages, spawning seasonality and length at sexual maturity. Journal of Applied Ichthyology, 25(1), 18-25.

Reis-Filho, J.A., \& de Alcântara Santos, A.C. (2014). Effects of substratum type on fish assemblages in shallow areas of a tropical estuary. Marine Ecology, 35(4), 456-470.

Robertson, D.R., \& Allen, G.R. (2015). Peces costeros del Pacifico Oriental Tropical: sistema de información en línea. Recuperado de http://biogeodb.stri.si.edu/ sftep/es/pages

Rodríguez-Romero, J., López-González, L., Galván-Magaña, F., Sánchez-Gutiérrez, F.J., Inohuye-Rivera, R.B., \& Pérez-Urbiola, J.C. (2011). Seasonal changes in a fish assemblage associated with mangroves in a coastal lagoon of Baja California Sur, Mexico. Latin American Journal of Aquatic Research, 39(2), 250-260.

Rodríguez-Climent, S., Caiola, N., \& Ibáñez, C. (2013). Salinity as the main factor structuring small-bodied fish assemblages in hydrologically altered Mediterranean coastal lagoons. Scientia Marina, 77(1), 37-45.

Romero-Berny, E.I., Velázquez-Velázquez, E., Schmitter-Soto, J.J., \& Salgado-Ugarte, I.H. (2020). The influence of habitat on the spatio-temporal dynamics of fish assemblages in a lagoon-estuarine system of the Mexican Pacific. Latin American Journal of Aquatic Research, 48(1), 23-37.

Ruiz-Ramírez, S., Molina-Arenas, E.G., Lucano-Ramírez, G., Aguilar-Betancourt, C., Flores-Ortega, J.R., Kosonoy-Aceves, D., \& González-Sansón, G. (2017). Aspectos reproductivos de la lisa Mugil curema (Mugiliformes: Mugilidae) en la laguna costera de Barra de Navidad, Jalisco, México. Latin American Journal of Aquatic Research, 45(2), 443-456.

Selleslagh, J., Amara, R., Laffargue, P., Lesourd, S., Lepage, M., \& Girardin, M. (2009). Fish composition and assemblage structure in three Eastern English Channel macrotidal estuaries: a comparison with other French estuaries. Estuarine, Coastal and Shelf Science, 81(2), 149-159.

Sheaves, M., \& Johnston, R. (2009). Ecological drivers of spatial variability among fish fauna of 21 
tropical Australian estuaries. Marine Ecology Progress Series, 385, 245-260.

Sosa-López, A., Mouillot, D., Ramos-Miranda, J., FloresHernandez, D., \& Chi, T.D. (2007). Fish species richness decreases with salinity in tropical coastal lagoons. Journal of Biogeography, 34(1), 52-61.

ter Braak, C., \& Smilauer, P. (2002). Canoco Reference Manual and Canodraw for Windows User's Guide: Software for Canonical Community Ordination. Versión 4.5. Ithaca, New York, USA: Microcomputer power.

Van Colen, C., Verbelen, D., Devos, K., Agten, L., Van Tomme, J., Vincx, M., \& Degraer, S. (2014). Sediment-benthos relationships as a tool to assist in conservation practices in a coastal lagoon subjected to sediment change. Biodiversity and Conservation, 23(4), 877-889.

Van der Wal, D., Herman, P.M.J., Forster, R.M., Ysebaert, T., Rossi, F., Knaeps, E. ... Ides, S.J. (2008). Distribution and dynamics of intertidal macrobenthos predicted from remote sensing: response to microphytobenthos and environment. Marine Ecology Progress Series, 367, 57-72.

Vinagre, C., Fonseca, V., Cabral, H., \& Costa, M.J. (2006). Habitat suitability index models for the juvenile soles, Solea solea and Solea senegalensis, in the Tagus estuary: defining variables for species management. Fisheries Research, 82(1-3), 140-149. 